Revista de Matemática: Teoría y Aplicaciones 2001 8(1) : 47-62

CIMPA - UCR - CCSS ISSN: 1409-2433

\title{
ON THE DISTRIBUTION OF NON-ATTACKING BISHOPS ON A CHESSBOARD C
}

\author{
SHANAZ ANSARI WAHID*
}

Received: 24 September 1999

\begin{abstract}
It is shown how the placement of non-attacking bishops on a chessboard $C$ is related to the matching polynomial of a bipartite graph. Reduction algorithms for finding the bishop polynomial of $C$ are given. We interpret combinatorially the coefficients of this polynomial and construct some interesting boards. Some applications of the bishop polynomials are given.
\end{abstract}

Keywords: bishop polynomials, bipartite graphs, matching, chessboard.

\section{Resumen}

Se muestra cómo la colocación de alfiles que no atacan en un tablero de ajedrez $C$ se relaciona con el polinomio de apareamiento de un grafo bipartito. Se dan algoritmos de reducción para encontrar el polinomio del alfil de $C$. Se interpretan combinatoriamente los coeficientes de este polinomio y se construyen algunos tableros interesantes. Se dan algunas aplicaciones de los polinomios de alfiles.

Palabras clave: polinomios de alfiles, grafos bipartitos, apareamiento, tablero de ajedrez.

Mathematics Subject Classification: 68R10.

\section{Introduction}

The graphs considered here are finite, undirected and have neither loops nor multiple edges. Let $G$ be a graph with $p$ nodes. A matching $M$ of $G$ is a spanning subgraph

${ }^{*}$ Centre for Combinatorics, Department of Mathematics and Computing, The University of the West Indies, St. Augustine, Trinidad. E-Mail: shanazw@yahoo.com 
whose components are nodes and edges only. A $k$-matching is a matching with $k$ edges and therefore $p-2 k$ isolated nodes. We will denote the number of $k$-matchings in $G$ by $m(G, k)$ or $a_{k}$. It is clear that $m(G, k)$ is equal to the number of selections of $k$ independent edges in $G$. A perfect matching consists of edges only.

The matching polynomial of a graph $G$ was originally defined in Farrell [1]. Here we assign to each node and edge of $G$, the weight $w_{1}$ and $w_{2}$ respectively. The matching polynomial of $G$ is

$$
M\left(G ; w_{1}, w_{2}\right)=\sum w(M)
$$

where the summation is taken over all the matchings $M$ in $G$. This can also be written as

$$
M\left(G ; w_{1}, w_{2}\right)=\sum_{k=0}^{\left[\frac{p}{2}\right]} m(G, k) w_{1}^{p-2 k} w_{2}^{k}
$$

Two graphs are said to be comatching if they have the same matching polynomial. The complement of a graph $G$ is normally taken with respect to the complete graph $K_{p}$ and is written as $\bar{G}$. The edges of $\bar{G}$ are those edges of $K_{p}$ which are not found in $G$. In some cases complement can be taken with respect to the complete bi-partite graph $K_{m, n}$.

In this paper we define the bishop polynomial of a chessboard $C$ as $\sum b_{k} x^{k}$, where $b_{k}$ is the number of ways placing $k$ non-taking bishops on $C$. Two chessboards $A$ and $C$ are said to be similar or equivalent if they have the same bishop polynomial. We write board for the term chessboard.

$G_{C}$ is the associated bi-partite graph for finding the bishop polynomial of $C$. The construction of $G_{C}$ is described in the next section. There are two types of diagonals described in $C$. The ith right diagonal with positive gradient is written as $r_{i}$ and the $j$-th left diagonal with negative gradient is written as $l_{j}$. A cell $e$ is uniquely described by the intersection of two diagonals $r_{i}$ and $l_{j}$ of $C$ and can be written as $e=(i, j)$. The board $C-e$ is obtained by removing cell $e$ from $C$ and the board $C-[e]$ is obtained by removing all the cells of the two diagonals (left and right) that intersect at $e$. The board $C-r_{i}$ is obtained from $C$ by removing the right diagonal $r_{i}$. Similarly, the board $C-l_{i}$ is obtained from $C$ by removing the right diagonal $l_{i}$.

When we say that a chessboard has $m$ rows and $n$ columns, we mean that each of these rows and each of these columns contains at least one cell. A cell in a board is depicted as an ' $x$ '. By disjoint boards $C_{1}$ and $C_{2}$ we mean boards which do not contain cells in the same left diagonal or right diagonal.

\section{The associated graph $G_{C}$ for $C$}

For each chessboard $C$ with $m$ rows and $n$ columns we refer to an associated rectangular $(m \times n)$ board. The diagonals $r_{i}$ are ordered from the $(1,1)$-th position along the first row of $C$ until $(1, n)$-th position, i.e. the end of first row. It then continues to the $(m, n)$ th position via column $n$. Thus diagonals $r_{i}$ exists for $i=1,2, \ldots, m+n-1$. The diagonals $l_{i}$ are measured from the $(m, 1)$-th position of first column and proceeds sequentially to 

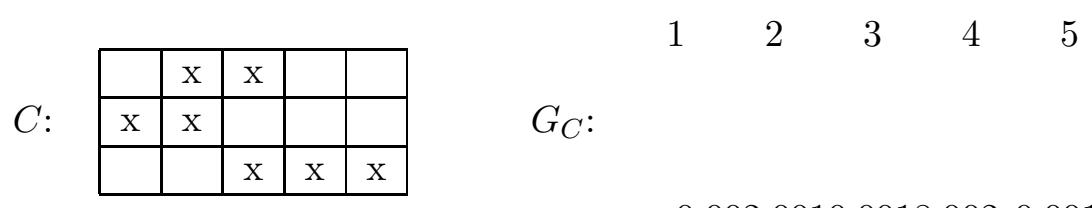

10.002:010.6018.0Q2 0.0018.00320.002.004 20.002.005

Figure 1: The construction of a bipartite graph for a given board.

the $(1,1)$-th position of the first column. We then proceed along first row to reach the $(1, n)$-th position, i.e end of first row. Thus diagonals $l_{i}$ exists for $i=1,2, \ldots, m+n-1$.

With the board $C$ we associate a graph $G_{C}$ as follows. The set of right diagonals give rise to a set of nodes $V_{1}$ and the set of left diagonals give rise to a set of nodes $V_{2}$ which are each labelled $1,2, \ldots, m+n-1$. An edge vivj is drawn where $v_{i} \in V_{1}$ and $v_{j} \in V_{2}$ iff there is a cell $(i, j)$ in $C$. Since a cell in $C$ is on the intersection of a right diagonal and a left diagonal, the graph $G_{C}$ is bipartite. We also note that a diagonal may contain no cells of $C$ and thus we ignore such a diagonal when drawing $G_{C}$. We now illustrate the construction of $G_{C}$ for a board $C$.

Example 1. Find $G_{C}$ for a board $C$ shown below.

Solution: There are 5 right diagonals since 2 (1st and 4 th) of the 7 right diagonals have no cells. There are 4 left diagonals since 3 (first, 6th and 7th) of the 7 left diagonals are empty. The 2 cells are listed from row 1 as $(1,3)$ and $(2,4)$. The two cells of second row are $(1,1)$ and $(2,2)$. The three cells of the third row are $(3,2),(4,3)$ and $(5,4)$. We then draw the bipartite graph $G_{C}$ as shown above in Figure 1. $G_{C}$ is disconnected in agreement with the fact that $C$ consists of two subboards: $V_{1}=\{1,2,3,4,5\}$ and $V_{2}=\{1,2,3,4\}$.

\section{Matchings and bishops}

Suppose that we choose cells from $G_{C}$ in such a way that if cell $(i, j)$ is selected, then no other cell in right diagonal $\mathrm{i}$ or left diagonal $\mathrm{j}$ can be chosen. Then we call such a choice of a cell as the assignment of $k$ non-attacking bishops on $G_{C}$. The following theorem is immediate.

Theorem $1 B(C ; x)=M\left(G_{C} ; 1, x\right)$.

Proof: Suppose that a bishop is placed on cell $(i, j)$ of $C$. Then no other bishop can be placed on that pair of diagonals that intersect on cell $(i, j)$ of $C$ in $G_{C}$. We note that cell $(i, j)$ is represented by the edge $v_{i} v_{j}$. The placing of a bishop on cell $(i, j)$ is equivalent to choosing the edge $v_{i} v_{j}$ of $G_{C}$ and ensuring that none of the other edges incident with vertices $i$ or $j$ are to be selected. Thus the distribution $k$ non-taking bishops $C$ is equivalent to a matching of $G_{C}$ with $k$ edges. It can be seen that every assignment of bishops corresponds to a unique matching in $G_{C}$ with $k$ edges and vice versa. The weight of an edge i.e. $w_{2}$ is taken as $x$ and that of a node is 1. 
We illustrate theorem 1 using the board $C$ shown before in Figure 1.

Example 2. The matching polynomial of the graph $G_{C}$ can be verified. $G_{C}$ consists of two disjoint trees on 4 nodes and 5 nodes. The matching polynomials are $w_{1}^{4}+3 w_{2} w_{1}^{2}+w_{2}^{2}$ and $w_{1}^{5}+4 w_{2} w_{1}^{3}+3 w_{2}^{2} w_{1}$.

$$
\begin{aligned}
M\left(G_{C} ; w_{1}, w_{2}\right) & =\left(w_{1}^{4}+3 w_{2} w_{1}^{2}+w_{2}^{2}\right)\left(w_{1}^{5}+4 w_{2} w_{1}^{3}+3 w_{2}^{2} w_{1}\right) \\
& =w_{1}^{9}+7 w_{2} w_{1}^{7}+16 w_{2}^{2} w_{1}^{5}+13 w_{2}^{3} w_{1}^{3}+3 w_{2}^{4} w_{1} . \\
B(C ; x) & =1+7 x+16 x^{2}+13 x^{3}+3 x^{4} .
\end{aligned}
$$

\section{Reduction algorithms for bishop polynomials}

The following theorem describes a reduction process for finding bishop polynomials.

Theorem 2 (The Fundamental Cell Theorem) $B(C ; x)=B(C-e ; x)+x B(C-$ $[e] ; x)$.

Proof: Let $e$ be a cell of $C$. Then we either put a bishop on $e$ or not. If a bishop is placed on $e$ then no other bishop can be placed on the two diagonals that intersect on $e$. Thus we remove the cells of the two diagonals that contain $e$. This gives the board $C-[e]$. We thus consider $B(C-[e] ; x)$ together with the factor $x$ which represents the contribution of one bishop on a cell $e$. If a bishop is not to be placed on cell $e$, then we remove this cell from $C$ and consider the board $C-e$. The result follows.

The following theorem gives the bishop polynomial of a board $C$ with $m$ disjoint subboards $C_{1}, C_{2}, \ldots, C_{k}$.

Theorem 3 (The Component Theorem) Let $B$ be a board consisting of $k$ disjoint sub-boards $C_{1}, C_{2}, \ldots, C_{k}$. Then $B(C ; x)=\prod_{i=1}^{k} B\left(C_{i} ; x\right)$.

Proof: The proof follows easily from the definition of disjoint sub-boards.

Theorem 4 (The Fundamental Right Diagonal Theorem) Let $C$ be a chessboard containing a right diagonal $r_{i}$ with $k$ cells. Let $C-r_{i, j}$ be the board obtained from $C$ by removing right diagonal $i$ and left diagonal $j$ (i.e. one of the $k$ left diagonals containing $a$ cell in the right diagonal $i$ ). Then

$$
B(C ; x)=B\left(C-r_{i} ; x\right)+x \sum_{j=1}^{k} B\left(C-r_{i, j} ; x\right) .
$$

Proof: We examine the distribution of bishops on $C$ with respect to the occupancy of the right diagonal $r_{i}$. If no bishop is placed on $r_{i}$ then we remove $r_{i}$ and consider the board $C-r_{i}$. If a bishop can be placed on a cell $e$ say, of $r_{i}$ then it can be any of the $k$ cells of $r_{i}$. No other bishop can be placed on $r_{i}$ and also the unique left diagonal $l_{j}$ which contained that particular cell $e$ of $r_{i}$. Thus we need to remove each of the $k$ left diagonals $l_{j}$ in turn 
with $r_{i}$. In so doing we consider the board. We multiply by x since one bishop has been placed on $r_{i}$. The result follows easily.

We give an illustration of Theorem 2. In the reduced boards, empty rows or columns that are situated between cells cannot be removed, else cells that were not in attacking positions may now be in attacking positions. It can be easily seen that if a board $C$ consists of a row of $k$ cells then this is similar to having $k$ disjoint sub-boards with polynomial $(1+x) k$.

Example 3. Find $B(C ; x)$ for the board $C$ in Example 1.

Solution: We reduce the board by the use of the fundamental cell algorithm as follows in Figure 2.

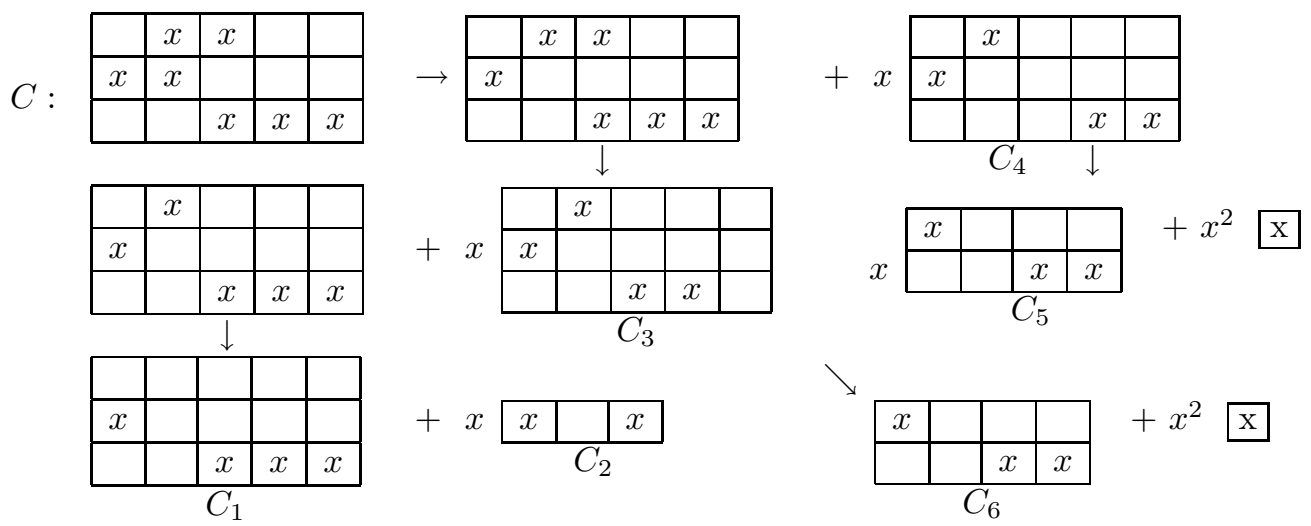

Figure 2: The reduction of a given board $C$.

At each stage of the reduction process, two boards are obtained. We get a board with one cell $e$ removed which is drawn first. The two diagonals that intersect on $e$ are then removed to give another board that is drawn second. The reduction process as described by Theorem 2 is applied repeatedly to all reduced boards.

If a row or column of a board has no cells, then we can remove the particular row or column before reducing the boards. However, we must ensure that in so doing, a cell $e$ which was not on the same diagonal as another cell $f$ say, is now placed on the same diagonal with $f$. This leads to an incorrect calculation of bishop polynomial.

$C_{1}$ consists of two disjoint subboards being a row of 1 cell and a row of 3 cells. $C_{6}$ consists of two disjoint subboards being a row of 1 cell and a row of 2 cells. $C_{6}$ and $C_{5}$ are similar. $C_{2}$ consists of a row of 2 cells. This gives by the component theorem the following polynomials:

$$
B\left(C_{1} ; x\right)=(1+x)^{4} ; B\left(C_{2} ; x\right)=(1+x)^{2} ; B\left(C_{5} ; x\right)=(1+x)^{3} .
$$

Thus we get

$$
\begin{aligned}
B(C ; x) & =B\left(C_{1} ; x\right)+x B\left(C_{2} ; x\right)+B\left(C_{6} ; x\right)+B\left(C_{5} ; x\right)+2 x^{2}(1+x) \\
& =1+7 x+16 x^{2}+13 x^{3}+3 x^{4} .
\end{aligned}
$$




\section{Coefficients of bishop polynomials}

A chessboard $C$ cannot be analyzed easily using combinatorics. It is advantageous to use the graph $G_{C}$ to find information about coefficients of $B(C ; x)$. We give expressions for the first four coefficients of the matching polynomial.

Lemma 1 Let $M\left(G ; w_{1}, w_{2}\right)=\sum_{k=0} a_{k} w_{2}^{k} w_{1}^{p-2 k}$. Then

(i) $a_{0}=1$.

(ii) $a_{1}=q$

(iii) $a_{2}=\left(\begin{array}{c}q \\ 2\end{array}\right)-\sum_{i=1}^{p}\left(\begin{array}{c}d_{i} \\ 2\end{array}\right)$ where $q$ is the number of edges of $G$ and $d_{i}$ is valency of node $i$.

(iv) $a_{3}=\left(\begin{array}{c}q \\ 3\end{array}\right)-(q-2) \sum_{i=1}^{p}\left(\begin{array}{c}d_{i} \\ 2\end{array}\right)+2 \sum_{i=1}^{p}\left(\begin{array}{c}d_{i} \\ 3\end{array}\right)+\sum_{i, j}\left(d_{i}-1\right)\left(d_{j}-1\right)-T$; where $T$ is number of triangles in $G$ and the last summation is done over all the edges ij in G, see Farrell et al. [3].

The following result of bishop polynomials is given noting that there are no triangles in a bipartite graph.

Theorem 5 Let the bishop polynomial $B(C ; x)$ of a chessboard $C$ be as defined. Let $C$ have $m$ right diagonals, $n$ left diagonals and $q$ cells. Then

(i) $b_{0}=1$.

(ii) $b_{1}=q$.

(iii) $b_{2}=\left(\begin{array}{c}q \\ 2\end{array}\right)-\sum_{i=1}^{m}\left(\begin{array}{c}d_{i} \\ 2\end{array}\right)-\sum_{j=1}^{n}\left(\begin{array}{c}d_{j} \\ 2\end{array}\right)$; where $d_{i}$ is number of cells in right diagonal $i$ and $d_{j}$ is the number of cells in left diagonal $j$.

(iv) $\begin{aligned} b_{3}= & \left(\begin{array}{c}q \\ 3\end{array}\right)-(q-2)\left\{\sum_{i=1}^{m}\left(\begin{array}{c}d_{i} \\ 2\end{array}\right)+\sum_{j=1}^{n}\left(\begin{array}{c}d_{j} \\ 2\end{array}\right)\right\} \\ & +2\left\{\sum_{i=1}^{m}\left(\begin{array}{c}d_{i} \\ 3\end{array}\right)+\sum_{j=1}^{n}\left(\begin{array}{c}d_{j} \\ 3\end{array}\right)\right\}+\sum_{i, j}\left(d_{i}-1\right)\left(d_{j}-1\right) \text {; where the last summation }\end{aligned}$ is done over all integer $i$ and $j$ for which the cell $(i, j)$ belongs to in $C$.

Proof: We need to examine the construction of $G_{C}$ from $C$. In $G_{C}$ a node corresponds to either a right or left diagonal. Also an edge of $G_{C}$ corresponds to a cell in $C$. Now 
the degree of a node in $G_{C}$ is the same as the number of cells in a right diagonal of left diagonal of $C$. Hence the results follows.

The following example illustrates theorem 5 .

Example 4. Find the first four coefficients of the bishop polynomial of the board $C$ in figure 1.

Solution: Clearly $b_{0}=1$. Now $C$ has 7 cells and thus $b_{1}=7$.

$$
\begin{aligned}
b_{2}= & \left(\begin{array}{l}
7 \\
2
\end{array}\right)-\left\{\left(\begin{array}{l}
2 \\
2
\end{array}\right)+\left(\begin{array}{l}
2 \\
2
\end{array}\right)\right\}-\left\{\left(\begin{array}{l}
2 \\
2
\end{array}\right)+\left(\begin{array}{l}
2 \\
2
\end{array}\right)+\left(\begin{array}{l}
2 \\
2
\end{array}\right)\right\} \\
= & 16 \\
b_{3}= & \left(\begin{array}{l}
7 \\
3
\end{array}\right)-(7-2)\left\{\left(\begin{array}{l}
2 \\
2
\end{array}\right)+\left(\begin{array}{l}
2 \\
2
\end{array}\right)+\left(\begin{array}{l}
2 \\
2
\end{array}\right)+\left(\begin{array}{l}
2 \\
2
\end{array}\right)+\left(\begin{array}{l}
2 \\
2
\end{array}\right)\right\}+0 \\
& +\{(2-1)(1-1)+(2-1)(2-1)+(2-1)(2-1)+(2-1)(2-1) \\
& +(1-0)(2-1)+(1-1)(2-1)+(1-1)(2-1)\} \\
= & 35-25+1+1+1 \\
= & 13
\end{aligned}
$$

\section{The use of $K_{m, n}$ in constructions of boards}

We give the construction of a few boards.

(a) Let $G_{C}$ be the complete bipartite graph $K_{m, n}$. $G_{C}$ has $m$ right diagonals and $n$ left diagonals. Since the graph is complete, then each right diagonal of board has $n$ cells and each left diagonal has $m$ cells. In this way there are $m n$ cells in all.

Example 5. Find $C$ for $K 3,5$.

Solution: We construct a board with 3 right diagonals and 5 left diagonals with no cells of a diagonal missing as shown below in Figure 3.

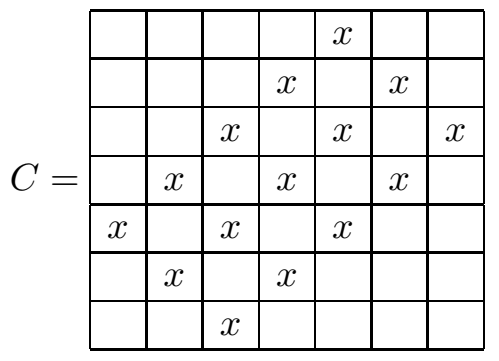

Figure 3: The chessboard for the complete bipartite graph $K_{3,5}$.

(b) We can use the chessboard for the complete bipartite graph $K_{m, n}$ to find $C$ for any bi-partite graph $G$. We need to identify which cells of the board corresponding to $K_{m, n}$ are to be used by examining the structure of the given graph $G$. This is shown 
for a path on $n$ nodes say. Let $G_{C}$ be a path on $n$ nodes ( $n$ is even). This path has an edge between the ith and $(i+1)$-th for $i=1,2, \ldots, n-1$. There are $n / 2$ nodes in each partition and thus $n / 2$ of both types of diagonals. Each node has degree 2 except the two end nodes. Thus the first right diagonal has one cell whilst all other right diagonals have 2 cells. Each left diagonal has 2 cells except the final one which has 1 cell.

Example 6. Find $C$ for the path $P_{n}$ on 8 nodes.

Solution: This bi-partite graph is shown below in Figure 4 . It has 7 edges. The nodes of the both sets are read equivalently as 1,2,3 and 4 . Thus the edge $(5,4)$ becomes $(3,2)$ say. The 7 cells are thus $(1,1),(2,1),(2,2),(3,2),(3,3),(4,3)$ and $(4,4)$ of the board corresponding to $K_{4,4}$. These are shown in Figure 4 .

$\begin{array}{llll}1 & 3 & 5 & 7 \\ 2 & 4 & 6 & 8\end{array}$

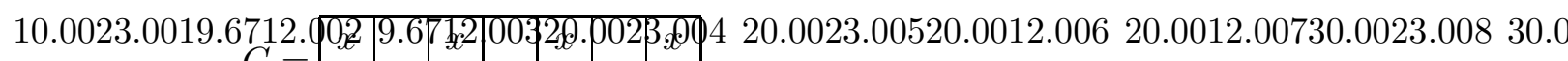

\begin{tabular}{llllllll|}
\hline & $=$ & $x$ & & $x$ & & $x$ & \\
\hline
\end{tabular}

Figure 4: The chessboard for for the path on 8 nodes.

(c) Furthermore, the unused cells of the board corresponding to $K_{m, n}$ constitute the board for the bipartite complement of $G$ i.e $\bar{G}$. Thus we can construct equivalent boards by using a pair of comatching bi-partite graphs. Firstly,the method described in (b) is applied to determine the board for each graph. Then the remaining cells of the two boards corresponding to $K_{m, n}$ gives the boards for the complements. An example of a comatching pair is shown in Figure 5. It has been proved, see Wahid [9], that the bipartite complements are also comatching. This next comatching pair leads to a second pair of equivalent boards. The following example illustrates the construction.

Example 7. Find the respective boards for the given graphs $G$ and $H$ as well as their complements as shown in Figure 5.

Solution: The given graphs are $G$ and $H$, which are then drawn with two disjoint node sets. Next we draw the bipartite complements $\bar{G}$ and $\bar{H}$. Finally both boards for each graph are drawn separately. The cells of the complements are shown with zeros. Use is made of the complete bipartite graph $K_{4,4}$ and its associated board. 


$\begin{array}{llll}1 & 3 & 5 & 7 \\ 2 & 4 & 6 & 8\end{array}$

$\bar{G}$

5.0053.0015.0042.002 5.0042.00315.0053.004 15.0053.00515.0042.006 15.0042.0072

\begin{tabular}{|l|l|l|l|l|l|l|}
\hline & & & 0 & & & \\
\hline & & 0 & & 0 & & \\
\hline & 0 & & 0 & & $x$ & \\
\hline$x$ & & $x$ & & $x$ & & $x$ \\
\hline & $x$ & & $x$ & & $x$ & \\
\hline & & 0 & & 0 & & \\
\hline & & & 0 & & & \\
\hline
\end{tabular}

$\overline{G_{C}}$

\begin{tabular}{|l|l|l|l|l|l|l|}
\hline & & & 0 & & & \\
\hline & & 0 & & $x$ & & \\
\hline & 0 & & 0 & & 0 & \\
\hline$x$ & & $x$ & & $x$ & & $x$ \\
\hline & $x$ & & $x$ & & $x$ & \\
\hline & & 0 & & 0 & & \\
\hline & & & 0 & & & \\
\hline
\end{tabular}

$\overline{H_{C}}$

Figure 5: A comatching pair of graphs, its complements and associated boards.

\section{Matchings and permutations in relation to non-taking bishops}

Let us consider the set $P$ of permutations of $n$ different elements with restrictions on their positions. We associate a graph $G_{P}$ as follows. The nodes of $G_{P}$ are in two disjoint sets $A$ and $B$ which represent the domain and range of the elements of $P$. We join two nodes $a$ and $b$ if and only if $b$ is a possible image of $a$. Thus $b$ is not a restricted position for $a$. Clearly $G_{P}$ is a bipartite graph. $G_{P}$ is not to be confused with $G_{C}$ since $C$ is a chessboard and $P$ is a permutation.

Let $\alpha$ be an element of $P$. Then $\alpha$ is represented by a subgraph $H_{\alpha}$ of GP defined as follows . The node set of $H_{\alpha}$ i.e. $V\left(H_{\alpha}\right)=V\left(G_{P}\right)$. Now domain $\alpha=A$ and range $\alpha=B$ and $H_{\alpha}$ is a spanning subgraph of $G_{P}$. Also, $a b$ is an edge of $H_{\alpha}$ if and only if $\alpha(a)=b$. Also $\alpha$ is $1-1$. Thus $H_{\alpha}$ is a perfect matching in $G_{P}$. 
Let $P_{k}$ be a set of $k$ independent edges in $G_{P}$. Then $P_{k}$ defines a permutation $\alpha_{k}$ of $k$ of the $n$ elements with the given restrictions. Thus if $P_{k}$ consists of the edges $a_{i_{1}}, b_{j_{1}}, a_{i_{2}}, b_{j_{2}}, \ldots, a_{i_{k}}, b_{j_{k}}$, then the corresponding permutation is $a_{i_{r}} \rightarrow b_{j_{r}}$, where $r=$ $1,2, \ldots, k$. Now every matching in $G_{P}$ having $k$ edges gives rise to a permutation $\alpha_{k}$ and conversely. Thus the number of permutations with restrictions of the $n$ elements taking $k$ at a time is equal to the number of matchings with $k$ edges in $G_{P}$.

In order to convert a problem to one of non-attacking bishops, we need to formulate an array which could then be used as an allowable chessboard $C$. For example, we examine the problem of placing $n$ different objects into $n$ distinct boxes so that no two objects occupy the same box. The answer is $n$ !.

A box can be treated as a cell. An actual distribution can be represented as a sequence $a_{i, j}$ meaning that object $i$ is placed in cell $j$. We first form an associated chessboard with the right diagonals indexed by the objects and the left diagonals indexed by properties of the objects. In this case there are $n$ right diagonals and $n$ left diagonals. The integer " 1 " is placed in position $(i, j)$ meaning that the $i$-th object is placed in cell $j$. Zeros are placed elsewhere. Since an object can occupy only one position, then each right diagonal must contain exactly one of " 1 ". Also no two objects can occupy the same cell and thus each left diagonal has exactly one of " 1 ". Clearly a "1" in the $(i, j)$ position means that no other " 1 " is in right diagonal $i$ or left diagonal $j$. This suggests the idea of non-taking bishops where a " 1 " is equivalent to a bishop. Thus the number of ways of placing $n$ distinct objects into distinct positions is the same as the number of ways of placing $n$ non-taking bishops on an $n$ by $n$ board $C$ as seen in Example 5 . It is easier to use the corresponding graph $K_{n, n}$ and extract the coefficient of $x^{n}$ with respect to Theorem 1 . The matching polynomial of $K_{n, n}$ is $\sum_{k} \frac{(m)_{k}(n)_{k}}{k !} w_{1}^{m+n-2 k} w_{2}^{k}$ with $k<\min (m, n)$, see Farrell [1]. The coefficient of $x^{n}$ is $\frac{(n)_{n}(n)_{n}}{n !}$ This is $n$ !.

Certain problems have restrictions on the placement of the bishops and this leads to the forbidden chessboard $C^{*}$. This board may have fewer cells than $C$ and can be easier to work with.

The Principle of Inclusion and Exclusion can be used to prove the following Theorem with respect to $C^{*}$.

Theorem 6 The number of permutations of $n$ objects in which no object is in a restricted position is $\sum_{k=0}^{n}(-1)^{k} b_{k}\left(C^{*}\right)(n-k)$ !, where $C^{*}$ is the board of forbidden cells.

Proof: Consider the set of permutations of $n$ objects with restrictions on their positions. Let $a_{i}$ be the property that the $i$-th object is in a forbidden position. Then we seek to find $N\left(a_{1}^{\prime}, a_{2}^{\prime}, \ldots, a_{n}^{\prime}\right)$. This is expanded as

$$
N\left(a_{1}^{\prime}, a_{2}^{\prime}, \ldots, a_{n}^{\prime}\right)=N-\sum_{i=1}^{n} N\left(a_{i}\right)+\sum_{1 \leq i \neq j \leq n} N\left(a_{i} a_{j}\right)-\ldots(-1)^{n} N\left(a_{1} a_{2} \cdots a_{n}\right)
$$

The number of permutations with propery $a_{i}$, i.e. $N\left(a_{i}\right)$ is found as follows. We need to place the $i$-th object in its forbidden position. This is equal to the number of ways of 
placing one non-taking bishop on $C^{*}$, i.e. $b_{1}\left(C^{*}\right)$. The other $n-1$ objects can be placed without restrictions in $(n-1)$ ! ways. Thus

$$
\sum_{i} N\left(a_{i}\right)=b_{1}\left(C^{*}\right)(n-1) !
$$

In general $N\left(a_{1} a_{2} \cdots a_{k}\right)$ is the number of permutations in which objects $1,2, \ldots, k$ are in their forbidden positions. Now $k$ of the $n$ objects can be placed in the forbidden positions in $b_{k}\left(C^{*}\right)$ ways and the remaining $n-k$ objects are permuted in $(n-k)$ ! ways. Thus

$$
\sum N\left(a_{1} a_{2} \cdots a_{k}\right)=b_{k}\left(C^{*}\right)(n-k) !
$$

The result follows on summing the contributions.

\section{Research developments and applications}

We examine some areas of study by means of the graph-theoretic approach to bishop theory.

\section{(a) Guarding bishops}

Suppose there is a system of diagonal corridors as shown in Figure 3 which has to be guarded by robots. The cells indicate important areas of the grid.The role of a robot can be viewed as that of a bishop. We simply place three non-attacking bishops since there are 3 right diagonals, noting that the robots must not attack one another. It is possible that a certain cell may be guarded by at least one bishop.

\section{(b) Discordant permutations}

Definition 1 Two permutations $\alpha_{1}$ and $\alpha_{2}$ on $A=\{1,2, \ldots, n\}$ are said to be discordant when $\alpha_{1}(k) \neq \alpha_{1}(k)$ for all $k \in A$.

Theorem 7 The number of permutations that are discordant with the identity permutation based on $A$ is $n ! \sum_{k=0}^{n} \frac{(-1)^{k}}{k !}$.

Proof: If a permutation is discordant with the identity permutation, then every element must not be in the correct place i.e. $\alpha(k) \neq k$. for all $k \in A$. This type of permutation is called a derangement. This leads to the classical "problems de recontres" involves derangements, see Riordan [6]. This problem is similar to that of placing $n$ non-taking bishops on a chessboard as described in Example 5 corresponding to the complete bipartite graph $K_{n, n}$. The bishops are not to be placed on a cell $e=(k, k)$ for $k=1,2, \ldots, n$. These $n$ cells constitute the board $C^{*}$.It is the $n$-th row of cells of the general chessboard described in Example 5. It consists of $n$ disjoint subboards, each of which is a cell. By 
Theorem $4, B\left(C^{*} ; x\right)=(1+x)^{n}$. On expanding, the $k$-th term is $C(n, k)$. Thus on substituting into Theorem 6 , we get

$$
\sum_{k=0}^{n}(-1)^{k}\left(\begin{array}{c}
n \\
k
\end{array}\right)(n-k) !=\sum_{k=0}^{n}(-1)^{k} \frac{n !}{k !} .
$$

Similarly, we consider permutations that are discordant with two given permutations, i.e. two-discordant permutations. This is called the "problème des ménages", see Riordan $[6]$.

Theorem 8 The number of permutations that are discordant with the identity and the $n$-cycle $(123 \ldots n)$ is

$$
\sum_{k=0}^{n}(-1)^{k} \frac{2 n}{2 n-k}\left(\begin{array}{c}
2 n-k \\
k
\end{array}\right)(n-k) !
$$

Proof: In this case integer $\mathrm{i}$ is not in the ith and $(i+1)$-th position for $i=1,2, \ldots, n-1$ and $n$ is not in the first and last position. $C^{*}$ consists of the cells $(k, k),(k, k+1)$ for $k=1,2, \ldots, n-1$ and cells $(n, n),(n, 1)$. This board has $2 n$ cells. The graph $G_{C^{*}}$ is the circuit $C_{2 n}$ on $2 n$ nodes. The matching polynomial of this graph is well known, see Farrell [1] . We have

$$
M\left(C_{2 n} ; w_{1}, w_{2}\right)=\sum_{k=0}^{n} \frac{2 n}{2 n-k}\left(\begin{array}{c}
2 n-k \\
k
\end{array}\right) w_{1}^{2 n-2 k} w_{2}^{k}
$$

Hence $B\left(C^{*} ; x\right)=\sum_{k=0}^{n} \frac{2 n}{2 n-k}\left(\begin{array}{c}2 n-k \\ k\end{array}\right) x^{k}$.

The result follows by substituting into Theorem 6 .

We illustrate the board $C^{*}$ for $n=5$ in Figure 6 .

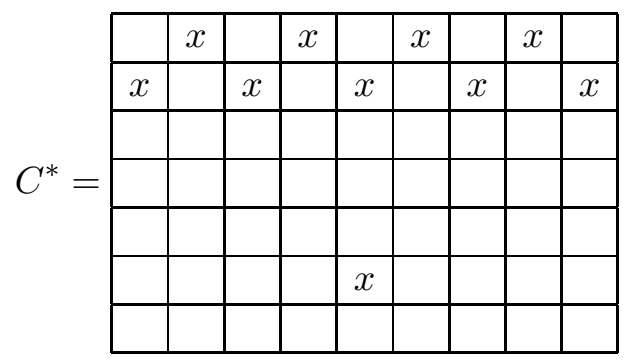

Figure 6: The board $C^{*}$ for $n=5$ in two discordant permutation.

In the same manner one can solve problems in $k$-discordant permutations for $k>2$. The calculations are tedious, even though it is better to work with the graph $G_{C^{*}}$ than the allowable board $C$. For a solution to three discordant permutations, see Farrell [2]. 


\section{(c)Bishop polynomials and determinants}

The following material has been reported in Wahid [8].

Definition 2 Let $G$ be a node labelled graph having $p$ nodes with labels $1,2, \ldots, p$. We associate with $G$, a $p \times p$ matrix $A(G)=\left(a_{i j}\right)$, where

$$
a_{i j}= \begin{cases}\sqrt{w_{2}} & \text { if nodes } i \text { and } j \text { are adjacent and } i<j \\ -\sqrt{w_{2}} & \text { if nodes } i \text { and } j \text { are adjacent and } i>j \\ 0 & \text { if nodes } i \text { and } j \text { are not adjacent } \\ w_{1} & \text { if } i=j .\end{cases}
$$

Then $A(G)$ is called the matching matrix of $G$. We associate a function called the $d$-function, i.e $d(A(G))$ on $A(G)$ as follows:

(i) For the null graph $d(A(G))=1$.

(ii) $d(A(G))=|A(G)|$, if $0<p<3$.

(iii) $d\left(A(G)=w_{1} d\left(A\left(G-v_{i}\right)\right)+w_{2} \sum_{v_{i} v_{j} \in E(G)} d\left(A\left(G-v_{i}-v_{j}\right)\right)\right.$; where $A\left(G-v_{i}\right)$ is the $(p-1) \times(p-1)$ matrix obtained from $A(G)$ by removing row $i$ and column $j$.

Lemma $2 d(A(G))=M\left(G ; w_{1}, w_{2}\right)$.

Proof: The result has been established in Wahid [8], by a proof using induction on the number of nodes in $G$.

Example 8. Let $G$ be the graph with edges $(1,2),(2,3),(2,4)$ and $(3,4)$. This is a triangle with a twig attached to node 1.

Then

$$
A(G)=\left[\begin{array}{cccc}
w_{1} & \sqrt{w_{2}} & 0 & 0 \\
-\sqrt{w_{2}} & w_{1} & \sqrt{w_{2}} & \sqrt{w_{2}} \\
0 & -\sqrt{w_{2}} & w_{1} & \sqrt{w_{2}} \\
0 & -\sqrt{w_{2}} & -\sqrt{w_{2}} & w_{1}
\end{array}\right]
$$

Using Lemma 2, we get

$$
\begin{aligned}
d(A(G)) & =M\left(G ; w_{1}, w_{2}\right) \\
& =w_{1} d\left[\begin{array}{ccc}
w_{1} & \sqrt{w_{2}} & \sqrt{w_{2}} \\
-\sqrt{w_{2}} & w_{1} & \sqrt{w_{2}} \\
-\sqrt{w_{2}} & -\sqrt{w_{2}} & w_{1}
\end{array}\right]+w_{2} d\left[\begin{array}{cc}
w_{1} & \sqrt{w_{2}} \\
-s q r t w_{2} & w_{1}
\end{array}\right] \\
& =w_{1}\left(w_{1}^{3}+3 w_{1} w_{2}\right)+w_{1}\left(w_{1}^{2}+w_{2}\right) \\
& =w_{1}^{4}+4 w_{1}^{2} w_{2}+w_{2}^{2}
\end{aligned}
$$


Also,

$$
\begin{aligned}
|A(G)| & =w_{1} d\left|\begin{array}{ccc}
w_{1} & \sqrt{w_{2}} & \sqrt{w_{2}} \\
-\sqrt{w_{2}} & w_{1} & \sqrt{w_{2}} \\
-\sqrt{w_{2}} & -\sqrt{w_{2}} & w_{1}
\end{array}\right|-\sqrt{w_{2}}\left|\begin{array}{ccc}
-s q r t w_{2} & \sqrt{w_{2}} & \sqrt{w_{2}} \\
0 & w_{1} & \sqrt{w_{2}} \\
0 & -\sqrt{w_{2}} & w_{1}
\end{array}\right| \\
& =w_{1}\left(w_{1}^{3}+3 w_{1} w_{2}\right)-\sqrt{w_{2}}\left(-\sqrt{w_{2}}\left(w_{1}^{2}+w_{2}\right)\right) \\
& =w_{1}^{4}+4 w_{1}^{2} w_{2}+w_{2}^{2} .
\end{aligned}
$$

Thus for some graph $G$, the matching function $d(A(G))$ gives the determinant. This is not true in general and is stated in the following theorem, see Wahid [8].

Theorem 9 Let $G$ be a graph with no circuits of even length. Then the matching polynomial of $G$ is the determinant of the matching matrix.

Definition 3 A d-graph of a graph $G$, denoted by $D(G)$ is a graph for which $|A(D(G))|=$ $M\left(G ; w_{1}, w_{2}\right)$.

From the definition, if a graph has no even cycles, then $G$ is same as $D(G)$. By finding $D\left(G_{C}\right)$ for the chessboard $C$, we proceed to the determinant by the above definition. This is stated as follows.

Theorem 10 Let $C$ be a board and $G_{C}$ its graph. Then $B(C ; x)=\left|A\left(D\left(G_{C}\right)\right)\right|$; where $w_{1}$ is replaced by $x$ and $w_{2}$ by 1 in the matching matrix.

The following example illustrates this theorem.

Example 9. Let $C$ and $G_{C}$ be as shown in Figure 1.

Since $G_{C}$ has no even cycles, $D\left(G_{C}\right)$ is identical to $G_{C}$. $G_{C}$ consists of two components, i.e. a path on 5 nodes and a path on 4 nodes. Thus we get by using Theorems 4 and 9 , the following product of two determinants:

$$
B(C ; x)=\left|\begin{array}{cccc}
1 & \sqrt{x} & 0 & 0 \\
-\sqrt{x} & 1 & \sqrt{x} & 0 \\
0 & -\sqrt{x} & 1 & \sqrt{x} \\
0 & 0 & -\sqrt{x} & 1
\end{array}\right| \times\left|\begin{array}{ccccc}
1 & \sqrt{x} & 0 & 0 & 0 \\
-\sqrt{x} & 1 & \sqrt{x} & 0 & 0 \\
0 & -\sqrt{x} & 1 & \sqrt{x} & 0 \\
0 & 0 & -\sqrt{x} & 1 & \sqrt{x} \\
0 & 0 & 0 & -\sqrt{x} & 1
\end{array}\right|
$$

These determinants can be easily evaluated.

Thus $B(C ; x)=\left(1+3 x+x^{2}\right)\left(1+4 x+3 x^{2}\right)=1+7 x+16 x^{2}+11 x^{3}+3 x^{4}$.

\section{(d) Connections with chromatic polynomials}

Definition 4 A proper colouring of the nodes of a graph $G$ is a colouring in which adjacent nodes are coloured differently. The chromatic polynomial of a graph $G$ is the number of proper colourings of the nodes with $\lambda$ colours. It is denoted as $P(G, \lambda)$. The basic properties of $P(G, \lambda)$ can be found in Read [5]. 
The matching polynomial is also related to the chromatic polynomial. The following lemma has been proved in Farrell and Whitehead [4].

Lemma 3 Let $M\left(G ; w^{\prime}\right)$ be the matching polynomial obtained by putting $w_{1}=w_{2}=w$. We then replace $w^{r}$ by $(\lambda)_{r}$ for all $r$ and use $(\lambda)_{r} \circ(\lambda)_{s}=(\lambda)_{r+s}$, where $\circ$ is the Zykov product. Then $M\left(G ; w^{\prime}\right)=P(\bar{G}, \lambda)$, if and only if $G$ has no triangles.

With respect to bishops, we need to consider graphs $G$ which can have circuits of even length. We then draw the board $C$ as stated in Section $6 . B(C ; x)$ is modified since the weights of $M\left(G ; w_{1}, w_{2}\right)$ are changed as seen in above Lemma 3 . We set $w_{1}=w_{2}=x$.

\section{Discussion}

It is not easy to analyse a chessboard combinatorially. It is easy to see that the graphs introduced give the problem of bishops a different outlook and makes it easier to solve. Bishop theory can be regarded as an application of matching theory and thus a closer connection between graph theory and combinatorics is seen.

The complete bipartite graph and its associated board plays an important role in the construction of boards and their complements. The algorithms described can be computerised to give efficient methods for finding $B(C ; x)$. The use of $B(C ; x)$ together with the Priciple of Inclusion and Exclusion is important for solving problems on permutations.

It should be noted that whenever recurrence relations are possible for matching polynomials of graphs like lattices or ladder type graphs, there would be corresponding recurrences for $B(C ; x)$. The use of $B(C ; x)$ for finding determinants and chromatic polynomials is quite interesting.

I am presently using $B(C ; x)$ to solve problems on polyominoes on $n$ cells without holes, see Sequence M1424, in Sloane and Plouffe [7]. This is an unsolved problem in mathematical physics. One hurdle is the question of symmetry in the graphs.

\section{References}

[1] Farrell,E.J. (1979) "An introduction to matching polynomials", J.of Comb. Theory B. 27: $75-86$.

[2] Farrell, E.J. (1998) "Three discordant permutations revisited", J. of Math. Sci. 9(1): 9-20.

[3] Farrell, E.J.; Guo, J.M.; Constantine, G.M. (1991) "On matching coefficients", Discrete Math. 89: 203-210.

[4] Farrell, E.J.; Whitehead, E.G. Jr.(1992) "Connections between the matching and chromatic polynomials", Internat. J. Math and Math. Sci. 15(4): 757-766.

[5] Read, R.C. (1968) "An introduction to chromatic polynomials", J. Combin. Theory 4: 52-71. 
[6] Riordan, J. (1980) An Introduction to Combinatorial Analysis. Princeton University Press, Princeton, New Jersey.

[7] Sloane, N.J.A.; Plouffe, S. (1995) The Encyclopedia of Integer Sequences. Academic Press, London and New York.

[8] Wahid, S.A. (1990) A Matrix Approach to Matching Polynomials. Ph.D. Thesis, University of the West Indies, St. Augustine, Trinidad, W.I.

[9] Wahid, S.A. (1983) On the Matching Polynomials of Graphs. M. Phil. Thesis, University of the West Indies, St. Augustine, Trinidad, W.I. 